\title{
Impulsividad en varones con prescripción de benzodiacepinas y metadona en prisión
}

\section{Impulsivity in men with prescription of benzodiazepines and methadone in prison}

\author{
Luis Moreno-Ramos*, María José Fernández-Serrano**, Miguel Pérez-García***, Antonio \\ VERDEJO-GARCÍA****. \\ *Centro Penitenciario de Albolote, Granada (España). **Departamento de Psicología. Universidad de Jaén. Jaén (España). \\ *** Centro de Investigación Mente, Cerebro y Comportamiento (CIMCYC). Universidad de Granada. Granada (España). \\ **** School of Psychological Sciences, Monash University. Melbourne (Australia).
}

\section{Resumen}

El consumo de benzodiacepinas y metadona se ha asociado a diversas alteraciones neuropsicológicas. Sin embargo, no conocemos estudios sobre el efecto de estas sustancias tanto de forma separada como de forma combinada en rasgos de personalidad impulsiva, y en menor medida en población penitenciaria. El objetivo principal de este estudio es examinar la impulsividad rasgo, medida con el Cuestionario de Sensibilidad al Castigo Sensibilidad a la Recompensa (Torrubia, Avila, Moltó y Caseras, 2001), y la escala de Evaluación del Comportamiento Impulsivo UPPS-P (Cyders et al., 2007), en una muestra de 134 varones de un centro penitenciario con consumo de metadona, metadona y benzodiacepinas, abstinentes de consumo, y no dependientes del consumo de sustancias (criterios DSM-IV). Los resultados mostraron que los grupos de consumidores presentan mayor sensibilidad a la recompensa, urgencia positiva, urgencia negativa y búsqueda de sensaciones que los no consumidores; los grupos de consumo de metadona presentan mayor sensibilidad al castigo y falta de perseverancia. El grupo de no consumidores presenta menor falta de perseverancia que el grupo de metadona y el grupo de metadona+benzodiacepinas. No se han encontrado diferencias específicamente del grupo de metadona+benzodiacepinas con el resto de los grupos. Como objetivo secundario, examinar, mediante análisis factorial exploratorio, qué dimensiones de personalidad impulsiva se relacionan con los dos sistemas motivacionales propuestos por Gray (SIC-SAC). Los resultados mostraron un componente definido por las subescalas sensibilidad al refuerzo, urgencia positiva, urgencia negativa y búsqueda de sensaciones, y un segundo definido por las subescalas sensibilidad al castigo, falta de perseverancia y falta de premeditación. Palabras clave: benzodiacepinas; metadona; impulsividad; prisión; UPPS-P; SCSR

\begin{abstract}
Benzodiazepines and methadone use has been associated with various neuropsychological impairments. However, to the best of our knowledge, no studies have been carried out on the effect of these substances (either separately or combined) on impulsive personality, including studies in prisoners. The aim of this study is to examine the impulsive personality of a sample of 134 male prisoners using the Sensitivity to Punishment and Sensitivity to Reward Questionnaire (Torrubia, Avila, Molto, \& Caseras, 2001) and the UPPS-P Scale (Cyders et al., 2007). Some of these were methadone users, methadone and benzodiazepines users, polydrug users in abstinence and non-dependent drug users. The results showed that drug users have greater sensitivity to reward, positive urgency, negative urgency and sensation seeking than non-dependent users. Methadone users showed more sensitivity to punishment and lack of perseverance with respect to other users. No differences were found between methadone+benzodiazepines users and other groups. The secondary aim is to examine which impulsive personality dimensions are related to the two motivational systems proposed by Gray (BISBAS) using exploratory factor analysis. Results showed two different components. One component was defined by the subscales sensitivity to reinforcement, positive urgency, negative urgency and sensation seeking. The second component was defined by the subscales sensitivity to punishment, lack of perseverance and lack of premeditation. Keywords: benzodiazepines; methadone; impulsivity; prison; UPPS-P; SPSRQ.
\end{abstract}


E 1 informe del Observatorio Europeo de las Drogas y las Toxicomanías (EMCDDA) de 2013 estima que la prevalencia media del consumo problemático de opiáceos entre adultos (de 15 a 64 años) es del $0,41 \%$, lo que supone 1,4 millones de casos en Europa para el año 2011. El 48\% de los individuos que iniciaron tratamiento por problema de adicciones en Europa para el año 2011 eran consumidores de opiáceos (principalmente heroína). En la literatura se encuentra gran cantidad de investigaciones que tienen por objeto estudiar la prevalencia y el efecto del consumo de estas drogas ilegales, siendo considerablemente menor la información que existe sobre el consumo de drogas prescritas. En este sentido, la metadona es el fármaco que más se prescribe, hasta en tres cuartas partes de los individuos con problemas de dependencia a opiáceos. Concretamente, en el contexto penitenciario español la prevalencia de tratamiento con metadona ronda el 7,9\% (2012) de la población interna (Secretaría General de Instituciones Penitenciarias, 2013). También en este contexto, la benzodiacepina se prescribía al 28,7\% de esta población (Subdirección General de Sanidad Penitenciaria, 2007). Además, la prevalencia del consumo de benzodiacepinas en pacientes que se encuentran en tratamiento con metadona se sitúa entre el $51 \%$ y el $70 \%$ (Jones, Mogali y Comer, 2012) y en España alrededor del 46,5\% (Fernández-Sobrino, Fernández-Rodriguez, y López-Castro, 2009). A pesar de estas prevalencias de consumidores de opiáceos y benzodiacepinas, existen relativamente pocos estudios sobre los efectos neuropsicológicos de estos fármacos, especialmente de las benzodiacepinas.

Las benzodiacepinas ejercen sus efectos a nivel cerebral a través de los receptores GABAA y su consumo ha sido asociado a alteraciones neuropsicológicas en la capacidad visuoespacial, velocidad de procesamiento y memoria verbal (Barker, Greenwood, Jackson, y Crowe, 2004; Stewart, 2005). Asimismo, algunos estudios en consumidores de esta sustancia han encontrado alteraciones en la impulsividad indicando comportamientos desinhibidos (Michel y Lang, 2003), toma de decisiones impulsivas (Dassanayake et al., 2012; Lane, Tcheremissine, Lieving, Nouvion, y Cherek, 2005), y déficits en la inhibición de respuesta (Acheson, Reynolds, Richards, y de Wit, 2006).

Distintos modelos teóricos sobre adicción han señalado la impulsividad como un marcador de vulnerabilidad de gran relevancia en la explicación de los procesos adictivos, tanto para explicar el inicio como el mantenimiento del consumo de drogas (Adan, 2002, Arce y Santisteban, 2006; Cano-Cervantes, Araque-Serrano, y Cándido-Ortiz, 2011; Cortés-Tomás, Giménez-Costa, Motos-Sellés, y Cadaveira-Mahía, 2014; Gullo, Loxton, y Dawe, 2014; Navas, Torres, Cándido, y Perales, 2014; Pattij y De Vries, 2013). El estudio de la impulsividad ha venido marcado por la utilización de al menos dos abordajes relativamente independientes: (i) estudio de la impulsividad cognitiva, a través del uso de pruebas neuropsicológicas y (ii) estudio de la im- pulsividad como rasgo de personalidad, a través del uso de medidas de autoinforme (Dougherty, Mathias, Marsh-Richard, Nouvion, y Dawes, 2008; Evenden, 1999; Perry y Carroll, 2008). Dentro de este segundo abordaje, existen dos propuestas teóricas fundamentales. Por un lado, Gullo et al. (2014) aportan evidencia de la existencia de dos factores para explicar la impulsividad en la conducta adictiva: "sensibilidad a la recompensa" ("reward sensitivity"), y una "impulsividad desinhibida" o "temeraria" ("rash impulsiveness”) (Dawe, Gullo y Loxton, 2004; Dawe, y Loxton, 2004; Franken y Muris, 2006). Por otro lado, Whiteside y Lynam (2001) y Cyders y Smith (2007) proponen el modelo de los cinco factores para explicar la personalidad impulsiva. En este modelo, explican que las cinco dimensiones que conforman la impulsividad son: la urgencia positiva, la urgencia negativa, la (falta de) premeditación, la (falta de) perseverancia y la búsqueda de sensaciones. Paralelamente a estos modelos, Gray y McNaughton (2000) en su modelo neuropsicológico de la personalidad proponen la existencia de dos sistemas motivacionales: el SAC (sistema de activación/ aproximación conductual) y el SIC (sistema de inhibición conductual). Mientras algunos trabajos han vinculado los dos factores de personalidad impulsiva propuestas por Gullo et al. con el sistema SAC (Dawe et al., 2004; Loxton et al., 2008a), otros trabajos apuntan que el sistema SAC estaría más vinculado a las dimensiones de urgencia y búsqueda de sensaciones, y el sistema SIC a la (falta de) premeditación y (falta de) perseverancia del modelo de los cinco factores (Verdejo-García et al., 2010a).

Tomando como base estos modelos teóricos, la literatura propone fundamentalmente dos instrumentos para la medición de la personalidad impulsiva. Por un lado Torrubia, Avila, Moltó y Caseras (2001) proponen el uso del "Cuestionario de Sensibilidad al Castigo Sensibilidad a la Recompensa" como un instrumento que permite la evaluación de dos dimensiones de personalidad: la sensibilidad al castigo (SC) y la sensibilidad a la recompensa (SR). Por otro lado, derivado de los análisis factoriales en los que se basa el modelo de los cinco factores, Whiteside y Lynam (2001) y Cyders et al. (2007) proponen el uso de la escala de Evaluación del Comportamiento Impulsivo UPPS-P. Carlson y Pritchard (2013) proponen que en la explicación de la conducta adictiva resulta más conveniente el uso combinado de la UPPS-P y el SCSR que cualquiera de ellas por separado.

Diversas investigaciones han estudiado la impulsividad como rasgo de personalidad en población dependiente o consumidora de sustancias. Globalmente los resultados de estos estudios indican una afectación de la personalidad impulsiva en consumidores de psicoestimulantes (Albein-Urios, Martínez-González, Lozano, Clark, y Verdejo-García, 2012; Fernández-Serrano et al., 2011; Verdejo-García et al., 2010a), consumidores intensivos semanales de alcohol (Motos, Cortés-Tomás, Giménez-Costa, y Cadaveira-Mahía, 2015), dependientes de alcohol (Bravo de Medina, Eche- 
burúa, y Aizpiri, 2007), dependientes de cannabis en tratamiento (Bravo de Medina, Echeburúa, y Aizpiri, 2010). Aunque el número de trabajos sobre opiáceos es menor, los resultados indican que también se podría producir una afectación de la personalidad impulsiva con respecto a individuos no dependientes del consumo de sustancias (Dissabandara, Loxton, Dias, Daglish, y Stadlin, 2012; Nielsen et al. 2012). Sin embargo, hasta donde llega nuestro conocimiento, no existen estudios sobre el efecto de las benzodiacepinas y la metadona tanto de forma separada como de forma combinada en rasgos de personalidad impulsiva, y en menor medida todavía en población penitenciaria.

Estudiar los rasgos de personalidad en población penitenciaria puede ser de gran interés por varios motivos. En primer lugar, por la alta prevalencia de uso de benzodiacepinas/sedantes de forma prescrita en este contexto (Subdirección General de Sanidad Penitenciaria, 2007). En segundo lugar, debido a que distintas investigaciones apuntan a la impulsividad como un factor de riesgo en la explicación de la conducta delictiva (Carroll et al., 2006; Mathias, Marsh-Richard, y Dougherty, 2008; Ratchford y Beaver, 2008), sería de interés estudiar qué dimensiones concretas se encuentran afectadas en esta población. Finalmente, por el propio interés que se deriva de la ausencia de investigaciones sobre este tema en este contexto, y por tanto, sus posibles implicaciones a nivel de prevención y tratamiento. Por estas razones, el objetivo principal de este estudio es examinar la personalidad impulsiva de pacientes con prescripción de benzodiacepinas en mantenimiento con metadona. Como objetivo secundario y tomando como base los modelos teóricos propuestos en la explicación de la impulsividad, en este estudio pretendemos examinar qué dimensiones de personalidad impulsiva medidas a través de la UPPS-P y la SCSR se relacionan con los dos sistemas motivacionales propuestos por Gray (SIC-SAC).

\section{Método}

\section{Participantes}

En esta investigación participaron 134 internos varones con edades comprendidas entre los 18 y los 50 años, procedentes del centro penitenciario de Albolote (Granada). La muestra se dividió en cuatro subgrupos, tres grupos de consumidores y uno de no dependientes del consumo de sustancias (en adelante abreviados como no consumidores), igualados en las variables de edad y años de escolarización (ver tabla 1). Dentro de los consumidores se contó con un grupo de metadona $(\mathrm{n}=33)$, un grupo de metadona+benzodiacepina $(n=29)$ y un grupo de policonsumidores dependientes abstinentes $(n=43)$. Todos ellos presentaban como droga preferente el consumo fumado de heroína y cocaína. Asimismo se contó con un cuarto subgrupo $(n=29)$ de individuos no dependientes del consumo de sustancias (criterios DSM-IV-TR., 2002).
El contexto en el que se lleva a cabo la investigación es un contexto penitenciario, por tanto se trata de un medio cerrado y con posibilidad reducida de acceso al consumo de otras drogas diferentes a las prescritas.

Fueron excluidos del estudio aquellos individuos que presentaran historia de daño cerebral traumático y alteraciones neurológicas y trastorno mental grave agudo medidas por entrevista.

\section{Instrumentos}

\section{Impulsividad como rasgo de personalidad.}

Escala de Evaluación del Comportamiento Impulsivo UPPS-P. (Whiteside y Lynam, 2001, adaptación española de Verdejo-García, Lozano, Moya, Alcázar, y Pérez-García, 2010b). Contiene 59 ítems, que miden cinco dimensiones de personalidad que pueden contribuir al comportamiento impulsivo: urgencia negativa, (falta de) perseverancia, (falta de) premeditación, búsqueda de sensaciones y urgencia positiva (Smith et al., 2007). La primera dimensión, urgencia negativa, evalúa la tendencia del individuo a ceder ante impulsos fuertes, específicamente cuando estos van acompañados de emociones negativas como depresión, ansiedad o ira. La segunda dimensión, (falta de) perseverancia, evalúa la habilidad del individuo para persistir en la realización de trabajos o en el cumplimento de obligaciones a pesar del aburrimiento o fatiga que puedan implicar. La tercera dimensión, (falta de) premeditación, evalúa la habilidad del individuo para pensar en las consecuencias potenciales de su comportamiento antes de llevarlo a cabo. La cuarta dimensión, búsqueda de sensaciones, evalúa la preferencia del individuo por la estimulación o excitación. La última dimensión, urgencia positiva, evalúa la tendencia del individuo a ceder ante impulsos cuando estos van precedidos de fuertes emociones positivas. Cada ítem era valorado siguiendo un formato tipo Likert, con 4 alternativas de respuesta, desde 1 (rotundamente de acuerdo) hasta 4 (rotundamente en desacuerdo). Se utilizó la puntuación total de cada una de las cinco dimensiones en los correspondientes análisis estadísticos.

Cuestionario de Sensibilidad al Castigo y Sensibilidad a la Recompensa (SCSR). (Torrubia et al., 2001). Es un cuestionario de 48 ítems que evalúa dos dimensiones de personalidad ortogonales: la sensibilidad al castigo (SC) y la sensibilidad a la recompensa (SR). Estas escalas evalúan diferencias individuales en la actividad del Sistema de Inhibición Conductual (SIC) y del Sistema de Activación Conductual (SAC) del modelo de personalidad neuropsicológico de Gray (Gray y McNaughton, 2000). El primer sistema toma el control de la conducta en respuesta a señales de castigo, no recompensa y estimulación nueva, y está relacionado con la dimensión ansiedad (rasgo). El segundo sistema implica un acercamiento conductual en respuesta a señales de recompensa o no castigo y está relacionado con la dimensión de impulsividad de 
personalidad. Las escalas SC y SR poseen adecuadas propiedades psicométricas según distintos estudios (Caseras, Avila y Torrubia, 2003; Verdejo et al., 2010b).

\section{Procedimiento}

El estudio cuenta con el visto bueno de la Dirección General de Instituciones Penitenciarias para la realización de estudios de investigación en centros penitenciarios.

Los individuos participantes en este estudio fueron reclutados mediante contactos individuales y a través de carteles informativos en los distintos módulos del centro penitenciario. Después de informar de los objetivos de la investigación, haciendo énfasis en que la participación en el estudio no tendría repercusión penitenciaria alguna para el individuo de forma que aumentase la fiabilidad de la información proporcionada, firmaban una hoja de consentimiento informado y recibían una compensación monetaria de 18 euros por su colaboración y la posibilidad de recibir un informe de los resultados.

Los participantes fueron evaluados tanto individualmente como colectivamente, siendo los instrumentos parte de un protocolo mayor dirigido a evaluar el rendimiento neuropsicológico de la muestra.

\section{Análisis estadísticos}

En primer lugar para comprobar la existencia de posibles diferencias entre los grupos en las variables de edad y años de escolarización se llevaron a cabo análisis de varianza univariados (ANOVAs), y la prueba no paramétrica de Kruskal-Wallis para la variable edad. Para examinar posibles diferencias entre los cuatro grupos en UPPS-P y SCSR se realizaron en primer lugar dos análisis multivariados de varianza (MANOVAs), y en segundo lugar, para los resultados estadísticamente significativos de los MANOVAs, se realizaron ANOVAs univariados post hoc para cada una de las dimensiones de las dos pruebas en las que hubiéramos obtenido resultados significativos. Seguidamente se realizaron análisis post hoc (prueba de Tuckey) para examinar la existencia de posibles diferencias entre los cuatro grupos en las diferentes dimensiones de las dos pruebas. Asimismo, se calculó el tamaño del efecto de las diferencias grupales en las variables dependientes utilizando $d$ de Cohen. Se fija el valor significación estadística del $5 \%$. Finalmente, con objeto de probar si se mantiene la estructura de componentes principales de impulsividad, se llevó a cabo un análisis factorial exploratorio con el método de extracción componentes principales y rotación varimax. Se extrajeron componentes con autovalor mayor que 1 .

\section{Resultados}

Respecto a las variables sociodemográficas los resultados mostraron que no existían diferencias estadísticamente significativas en escolaridad, En cuanto a la edad, no se distribuía normalmente, por lo que se procedió a realizar la prueba no paramétrica de Kruskal-Wallis (ver Tabla 1)

A continuación se procedió a estudiar las posibles diferencias de los grupos en las variables dependientes relacionadas con personalidad impulsiva (UPPS-P y SCSR).

En primer lugar el MANOVA entre grupos para la tarea SCSR mostró diferencias estadísticamente significativas entre los grupos, Lambda de Wilks, [F $(6,258)=5,852 ; \mathrm{p}<.001$, $\left.\eta^{2}=.12\right]$. Seguidamente se realizó ANOVAs univariados post hoc para las dos subescalas. Los resultados mostraron efectos significativos en SC, $\left[\mathrm{F}(3,130)=3,481 ; \mathrm{p}=.018, \eta^{2}=\right.$ $.07]$ y en SR, $\left[\mathrm{F}(3,130)=9,528 ; \mathrm{p}<.001, \eta^{2}=.18\right]$. Los análisis post hoc de los ANOVAS univariados indicaron que para la subescala SC sólo existen diferencias estadísticamente significativas entre el grupo de no consumidores y el grupo de metadona $(\mathrm{p}=.034)$. Para la subescala SR los resultados mostraron diferencias estadísticamente significativas entre el grupo de no consumidores y el resto de grupos $(\mathrm{p}<.001$ en todas las comparaciones). Los tamaños del efecto (delta de Cohen) obtenidos fueron entre medios y altos para todas las comparaciones (valores entre .74 y 1.20) (ver tabla 2).

En segundo lugar el MANOVA entre grupos para la tarea UPPS-P mostró diferencias estadísticamente significativas entre los grupos, Lambda de Wilks, [F $(15,348)=$ 4,058; $\left.\mathrm{p}<.001, \eta^{2}=.14\right]$ Los ANOVAs univariados para las cinco subescalas mostraron efectos significativos en "urgencia positiva", [F $\left.(3,130)=9,058 ; \mathrm{p}<.001, \eta^{2}=.17\right]$ "urgencia negativa", [F $\left.(3,130)=13,273 ; \mathrm{p}<.001, \eta^{2}=.23\right]$ "búsqueda de sensaciones", [F $(3,130)=10,467 ; \mathrm{p}<.001, \eta^{2}=$ .19] y "falta de perseverancia", [F $(3,130)=5,655 ; \mathrm{p}=.001$, $\left.\eta^{2}=.11\right]$ No se obtuvieron resultaron significativos para "falta de premeditación", [F $(3,130)=1,396 ; p=.247, \eta^{2}=$ .03] . Los análisis post hoc de los ANOVAS univariados indicaron que para la subescalas "urgencia positiva",

Tabla 1. Puntuaciones descriptivas, comparaciones y significación de las características sociodemográficas de los grupos

\begin{tabular}{|c|c|c|c|c|c|c|}
\hline & Mt $(n=33)$ & $M t+B(n=29)$ & $A(n=43)$ & NDS $(n=29)$ & F/Chi cuadrado & $\mathbf{p}$ \\
\hline Edad & $36,06(4,64)$ & $34,96(4,54)$ & $31,88(8,62)$ & $34,57(7,25)$ & $5,69^{\star}$ & .128 \\
\hline Escolaridad & $7(2,23)$ & $7,48(1,66)$ & $7,55(1,85)$ & $8,14(1,86)$ & $1,79 * \star$ & .151 \\
\hline
\end{tabular}

Nota. $\mathrm{Mt}=$ metadona; $\mathrm{Mt}+\mathrm{B}=$ metadona+ benzodiacepinas; $\mathrm{A}=\mathrm{Abstinentes;} \mathrm{NDS}=$ no dependientes de sustancias; $\mathrm{M}=\mathrm{Media} ; \mathrm{DT}=\mathrm{Desviación}$ Típica ${ }^{*}=$ valor del estadístico chi cuadrado (kruskal-Wallis); ${ }^{* *}=$ valor del estadístico $\mathrm{F}$. 
"urgencia negativa", "búsqueda de sensaciones" existían diferencias estadísticamente significativas entre el grupo de no consumidores y el resto de grupos (metadona, metadona+ benzodiacepinas y abstinentes) con valores $\mathrm{p} \leq .005$. Los delta de Cohen obtenidos fueron altos para todas las comparaciones (valores entre 1.01 y 1.58 ). Para la subescala "falta de perseverancia" los resultados mostraron diferencias estadísticamente significativas entre el grupo de no consumidores respecto al grupo de metadona y el grupo de metadona+benzodiacepinas $(\mathrm{p} \leq .017)$. Los tamaños de efecto obtenidos fueron entre medios y altos $(0.72$ y 0.99$)$. (Ver tabla 2)

Tabla 2. Puntuaciones descriptivas de los cuatro grupos en las distintas dimensiones de la UPPS-P y escalas de SCSR, clasificados en función de los dos componentes obtenidos junto con tamaños del efecto (delta de Cohen) de las comparaciones entre pares de grupos

\begin{tabular}{|c|c|c|c|c|c|c|}
\hline Instrumentos & $\begin{array}{l}M t(n=33) \\
M(D T)\end{array}$ & $\begin{array}{l}M t+B(n=29) \\
M(D T)\end{array}$ & $\begin{array}{l}A(n=43) \\
M(D T)\end{array}$ & $\begin{array}{l}\text { NDS }(n=29) \\
M(D T)\end{array}$ & Tuckey & d \\
\hline \multicolumn{7}{|l|}{ SCSR } \\
\hline SC & $13,84(4,62)$ & $12,75(5,96)$ & $10,76(5,19)$ & $10,17(5,23)$ & $\mathrm{Mt}\rangle \mathrm{A}=\mathrm{NDS}$ & 0,74 (Mt-NDS) \\
\hline \multirow[t]{3}{*}{ SR } & $12,60(3,91)$ & $12,51(4,38)$ & $12,46(4,38)$ & $7,79(4,12)$ & $N D S<M t=M t+B=A$ & $1,20($ Mt-NDS) \\
\hline & & & & & & $1,11(\mathrm{Mt}+\mathrm{B}-\mathrm{NDS})$ \\
\hline & & & & & & 1,09(A-NDS) \\
\hline \multicolumn{7}{|l|}{ UPPS-P } \\
\hline \multirow[t]{3}{*}{ Urgencia positiva } & $32,69(8,37)$ & $34,41(9,39)$ & $30,69(6,93)$ & $23,96(8,63)$ & $\mathrm{NDS}<\mathrm{Mt}=\mathrm{Mt}+\mathrm{B}=\mathrm{A}$ & $1,02(\mathrm{Mt}-\mathrm{NDS})$ \\
\hline & & & & & & $1,15(\mathrm{Mt}+\mathrm{B}-\mathrm{NDS})$ \\
\hline & & & & & & 1,01 (A-NDS) \\
\hline \multirow[t]{3}{*}{ Urgencia negativa } & $31,33(6,87)$ & $34,20(6,72)$ & $30,51(6,10)$ & $23,86(6,33)$ & $N D S<M t=M t+B=A$ & 1,12(Mt-NDS) \\
\hline & & & & & & $1,58(\mathrm{Mt}+\mathrm{B}-\mathrm{NDS})$ \\
\hline & & & & & & 1,07(A-NDS) \\
\hline \multirow{3}{*}{$\begin{array}{l}\text { Búsqueda de } \\
\text { sensaciones }\end{array}$} & $32,12(7,08)$ & $32,75(7,94)$ & $33,23(5,33)$ & $24,96(7,02)$ & $N D S<M t=M t+B=A$ & $1,03(\mathrm{Mt}-\mathrm{NDS})$ \\
\hline & & & & & & $1,05(\mathrm{Mt}+\mathrm{B}-\mathrm{NDS})$ \\
\hline & & & & & & 1,38(A-NDS) \\
\hline \multirow{2}{*}{$\begin{array}{l}\text { Falta de } \\
\text { perseverancia }\end{array}$} & $21,42(4,67)$ & $20,58(5,44)$ & $19,20(3,30)$ & $17,24(3,57)$ & $N D S<M t=M t+B$ & 0,99(Mt-NDS) \\
\hline & & & & & & $0,72(\mathrm{Mt}+\mathrm{B}-\mathrm{NDS})$ \\
\hline $\begin{array}{l}\text { Falta de } \\
\text { premeditación }\end{array}$ & $21,96(5,23)$ & $22,41(5,90)$ & $21,55(4,20)$ & $19,67(4,95)$ & & \\
\hline
\end{tabular}

Nota. $\mathrm{Mt}=$ metadona; $\mathrm{Mt}+\mathrm{B}=$ metadona + benzodiacepinas; $\mathrm{A}=\mathrm{Abstinentes} ; \mathrm{NDS}=$ no dependientes de sustancias.

$\mathrm{SC}=$ Sensibilidad al Castigo; $\mathrm{SR}=$ Sensibilidad a la Recompensa; $M=$ Media; $\mathrm{DT}=$ Desviación Típica.

${ }^{*} d$ de Cohen $>.80$ indicando tamaño del efecto grande.

Tabla 3. Intercorrelaciones entre las distintas dimensiones del cuestionario SCSR y de la escala UPPS-P

\begin{tabular}{|c|c|c|c|c|c|c|c|}
\hline Dimensión & 1 & 2 & 3 & 4 & 5 & 6 & 7 \\
\hline 1. SC & ---- & & & & & & \\
\hline 2. $S R$ & $.236^{\star \star}$ & --- & & & & & \\
\hline $\begin{array}{l}\text { 3. Urgencia } \\
\text { negativa }\end{array}$ & $.366^{\star \star}$ & $.527^{\star \star}$ & ----- & & & & \\
\hline $\begin{array}{l}\text { 4. Falta de } \\
\text { premeditación }\end{array}$ & .077 & $.199^{\star}$ & $.428^{\star \star}$ & $----\cdot$ & & & \\
\hline $\begin{array}{l}\text { 5. Falta de } \\
\text { perseverancia }\end{array}$ & $.307^{\star \star}$ & $.341^{\star \star}$ & $.395^{\star \star}$ & $.599^{\star \star}$ & ------- & & \\
\hline $\begin{array}{l}\text { 6. Búsqueda de } \\
\text { Sensaciones }\end{array}$ & .029 & $.543^{\star \star}$ & $.460^{\star *}$ & $.181^{\star}$ & $.240^{\star \star}$ & ------- & \\
\hline $\begin{array}{l}\text { 7.Urgencia } \\
\text { positiva }\end{array}$ & $.342^{\star \star}$ & $.581^{\star \star}$ & $.759^{\star *}$ & $.439^{\star *}$ & $.481^{\star \star}$ & $.471^{\star *}$ & $\cdots$ \\
\hline
\end{tabular}

Nota. $\mathrm{N}=134 . \mathrm{SC}=$ Sensibilidad al Castigo; $\mathrm{SR}=$ Sensibilidad a la Recompensa. ${ }^{\star} p<.05 .{ }^{\star \star} p<.01$
Tabla 4. Cargas de los factores de la extracción de componentes principales con rotación varimax de las dimensiones de la escala UPPS-P y el Cuestionario SCSR.

\begin{tabular}{lccc}
\hline \multirow{2}{*}{ Dimensión } & \multicolumn{2}{c}{ Componentes } & \multirow{2}{*}{$\begin{array}{c}\text { Comunalidad } \\
\left(\mathbf{h}^{\mathbf{2}} \mathbf{)}\right.\end{array}$} \\
\cline { 2 - 3 } $\mathbf{S C}$ & $\mathbf{1}$ & $\mathbf{2}$ & .249 \\
\hline SR & 164 & .471 & .704 \\
\hline Urgencia negativa & .820 & .180 & .714 \\
\hline Falta de premeditación & .675 & .508 & .669 \\
\hline Falta de perseverancia & .069 &. $\mathbf{8 1 5}$ & .702 \\
\hline Búsqueda de sensaciones & .167 &. $\mathbf{8 3 2}$ & .700 \\
\hline Urgencia positiva & $\mathbf{8 3 7}$ & -.002 & .700 \\
\hline Porcentaje de varianza & $\mathbf{6 8 8}$ & $\mathbf{. 5 4 1}$ & \\
\hline Total porcentaje de varianza & 64,59 & & \\
\hline
\end{tabular}

Nota. Cargas de los factores $>.40$ están impresas en negrita. SC= Sensibilidad al Castigo; $\mathrm{SR}=$ Sensibilidad a la Recompensa. 
Finalmente, el análisis factorial exploratorio arrojó una solución de dos componentes principales con autovalores mayores de 1 (3.398 y 1.123 respectivamente), que explicaban en conjunto el $64.59 \%$ de la varianza total con un buen ajuste de la muestra (Kaiser-Meyer-Olkin $\mathrm{KMO}=.754$, Prueba de esfericidad de Barlett $\mathrm{p}<.001)$. El primer componente explica el $33.72 \%$ de la varianza y viene definido por las subescalas "SR", "urgencia positiva”, "urgencia negativa” y "búsqueda de sensaciones", con cargas factoriales mayores de .60. El segundo componente explica el $30.87 \%$ de la varianza. Viene definido por las subescalas "falta de perseverancia" y "falta de premeditación" con cargas mayores de .80, y la subescala "SC" con carga factorial de .47. La matriz de correlaciones aparece en la tabla 3. Las cargas factoriales de las subescalas en los dos componentes aparecen en la matriz de componentes rotados de la tabla 4.

\section{Discusión}

El objetivo principal de este estudio fue examinar la personalidad impulsiva de individuos penitenciarios en mantenimiento con metadona y prescripción de benzodiacepinas. Los resultados mostraron que los grupos de consumidores (metadona, metadona+benzodicepinas y abstinentes) mostraban mayor "sensibilidadala recompensa", "urgencia positiva", "urgencia negativa" y "búsqueda de sensaciones" que los convictos no-consumidores. También se ha encontrado que los grupos de consumo de metadona (metadona, metadona+benzodiacepinas) presentaban mayor "sensibilidad al castigo" y "falta de perseverancia". No se han encontrado diferencias específicamente del grupo de metadona+benzodiacepinas con el resto de los grupos. Por último, el análisis factorial exploratorio de los dos cuestionarios mostró un componente definido por las subescalas "SR", "Urgencia positiva", "Urgencia negativa" y "Búsqueda de Sensaciones," y un segundo definido por las subescalas SC, "Falta de perseverancia" y "Falta de premeditación”.

Las diferencias entre los grupos de consumo frente a no consumo en la urgencia positiva y negativa, la búsqueda de sensaciones y la SR podría indicar que estas características de personalidad están relacionadas de manera general con el consumo de drogas. Así, si bien en la literatura todas las escalas se han relacionado con la adicción, las dos escalas de "Urgencia” parecen mostrarse, más consistentes en la diferenciación de los grupos con dependencia/sin dependencia de sustancias (Verdejo-García et al., 2007, 2010a) denotando aspectos de inestabilidad emocional más propia de la mayor comorbilidad psicopatológica que presenta el grupo de consumidores frente a no consumidores (Billeux et al., 2012; Casares-López et al., 2011). En cuanto a la escala "SR", también ha sido consistentemente asociado al consumo (Balconi, Finocchiaro, y Campanella, 2014; Stautzy Cooper, 2013) tal vez por el hecho de que estaría relacionada con la vía mesolimbicocortical mediada por la transmisión dopaminérgica más sensibilizada en los consumidores (Robinson y Berridge, 1993). Por último, aunque la escala "Búsqueda de sensaciones" aparece más inconsistentemente relacionada en la literatura con la adicción (Verdejo-García et al., 2007, 2010a), en nuestro estudio también aparece como característica de la impulsividad asociada al consumo, posiblemente debido a que nuestros grupos de consumo presentan una mayor severidad de consumo y mayor frecuencia de implicarse en situaciones de riesgo que ello lleva asociado, pudiendo constituir además, un constructo temperamental característico penitenciario (Lykken, 1995).

Por otro lado, los grupos asociados al consumo de metadona (metadona y metadona+benzodicepinas) mostraron mayor falta de perseverancia y SC. Estos datos sugieren que el grupo de abstinentes poseería las características de tolerancia a la frustración y aburrimiento además de concentración en la tarea, que un proceso de deshabituación (con o sin apoyo especializado), requiere, y del que adolece los grupos con metadona más necesitados de un apoyo farmacológico. La metadona afecta procesos de atención selectiva (Mintzer y Stitzer, 2002; Prosser et al., 2006, y datos propios obtenidos en esta muestra y no publicados), y a su vez, estos procesos de inhibición de respuesta prepotente se han relacionado con la escala de "falta de perseverancia" (Cyders y Coskunpinar, 2011) por lo que se sugiere un proceso común subyacente especialmente distintivo en los grupos de consumo de metadona respecto al grupo de no consumidores. La mayor SC asociada a los grupos de metadona podría estar relacionado con una regulación a la baja de la actividad noradrenérgica debida a la estimulación crónica del receptor mu opioide que altera su procesamiento de percepción del castigo, tal como ha sido propuesta por Ersche et al. (2005).

Por último, respecto a las características de personalidad impulsivas de nuestros grupos debemos destacar que no se han encontrado diferencias entres ellos en la dimensión “(falta de) premeditación”. Esta escala ha sido relacionada con un proceso de toma de decisiones (Zermatten, Van der Linden, d'Acremont, Jermann, y Bechara, 2005) y es una predictora consistente de conductas externalizantes como la criminal (Gordon y Egan, 2011) o violencia general (Derifenko, DeWall, Metze, Walsh, y Lynam, 2011; Miller, Zeichner, y Wilson, 2012) por lo que podría considerarse como una dimensión de impulsividad común de los reclusos y no específica de la adicción.

Los resultados del objetivo secundario son parcialmente análogos a los de aquellos estudios (Mitchell et al., 2007; Perales, Verdejo-García, Moya, Lozano, y Pérez-García, 2009; Verdejo-García et al., 2010a) que apuntan que el sistema SAC estaría más vinculado a las dimensiones de urgencia y búsqueda de sensaciones, mientras que el sistema SIC estaría más vinculado, en base a su carga factorial, a las dimensiones falta de premeditación y falta 
de perseverancia. En nuestro estudio se observa que todos los sujetos consumidores de drogas, con independencia de la sustancia de consumo preferente, presentaban déficits en todas las escalas incluidas en el primer factor resultante del análisis por componentes, más vinculado al sistema SAC, en línea con distintas investigaciones que muestran que el sistema SAC juega un papel importante en la adicción a diferentes sustancias incluyendo heroína, metadona, cocaína, ketamina, alcohol y tabaco (Abdi, Roudsari, y Aliloo, 2011; Bijttebier, Beck, Claes, y Vandereycken, 2009; Carlson y Pritchard, 2013; Dissabandara et al., 2012, 2014; Franken, Muris, y Georgieva, 2006; Loxton et al., 2008a; Lyvers, Duff, Basch, y Edwards, 2012; Nielsen et al., 2012). Por otra parte, globalmente nuestros resultados indicarían que son los consumidores de metadona los que tendrían mayores déficits en las escalas incluidas en el segundo factor, más vinculado al sistema SIC. Si bien es cierto que la relación de SIC con el consumo de drogas no está tan bien establecida en la literatura como sucede con el SAC (Bijttebier et al. 2009; Dissabandara et al. 2012; Ersche et al. 2005).

Estos resultados tienen algunas implicaciones clínicas fundamentalmente de cara a la inclusión de la evaluación de los rasgos de impulsividad en el proceso de evaluación y tratamiento de los trastornos adictivos. En el caso de la evaluación, nuestros resultados apuntan a la posibilidad de elaboración de nuevos instrumentos de autoinformes teniendo en cuenta el solapamiento entre las escalas de UPPS, SR-SC y las dimensiones propuestas en el modelo de Gray. Respecto al tratamiento, Staiger, Kambouropoulos, y Dawe (2007) resaltan la importancia de desarrollar tratamientos específicos en función de los rasgos de personalidad evaluados. Por ejemplo, la terapia "manejo de contingencias" con pacientes con rasgos prominentes de "sensibilidad a la recompensa", entrenamiento en habilidades de resolución de conflictos, mindfullness, o terapia dialéctica conductual de Linehan para rasgos de "rash impulsivity" y estrategias cognitivo conductuales para rasgos de ansiedad comórbidos. Por último, recientemente se ha encontrado que las puntuaciones en las escalas "búsqueda de sensaciones" y "falta de premeditación" de la UPPS son potenciales moderadoras de los resultados de la terapia de aumento motivacional ("motivacional enhancement therapy") (Moshier, Ewen, y Otto, 2013).

Nuestro estudio presenta importantes fortalezas y algunas limitaciones. Entre las fortalezas destaca fundamentalmente el tipo de muestra utilizada, con un perfil de consumo de metadona y benzodiacepinas que nos permite conocer el efecto separado y combinado de estas sustancias sobre la personalidad impulsiva y que se encuentra además en contexto penitenciario. Respecto a las limitaciones, debemos destacar la ausencia de grupos de control no penitenciarios que pudieran evidenciar más claramente variables específicas al contexto criminológico y posibles diferencias entre grupos de consumidores. Por otro lado, nuestro estudio ha sido realizado sólo con muestra masculina. Si bien es cierto que la población masculina representa el $92,4 \%$ de la población reclusa española (Informe General 2012 de la Secretaría General de Instituciones Penitenciarias), sería interesante estudiar si estos resultados son extensibles a población reclusa femenina. Finalmente, las medidas de autoinforme, si bien eran el objeto de este estudio y se enmarcan en teorías comprehensivas, no abarcan de forma global el fenómeno complejo de la impulsividad. Sería interesante que futuros estudios se complementaran con otras medidas de impulsividad, tanto de autoinforme como de laboratorio.

\section{Reconocimientos}

Este trabajo ha sido financiado por una subvención P07. HUM 03089 de la Junta de Andalucía (Proyectos de excelencia 2007). Investigador principal: Miguel Pérez García.

\section{Conflictos de interés}

Los autores no tienen conflictos de intereses que declarar.

\section{Referencias}

Abdi, R., Roudsari, A. B., y Aliloo, M. M. (2011). The sensitivity level of behavioral approach and inhibition systems in substance abusers, smokers and normal subjets. Iranian Journal of Psychiatry and Clinical Psychology, 17, 241-247.

Acheson, A., Reynolds, B., Richards, J. B., y de Wit, H. (2006). Diazepam impairs behavioral inhibition but not delay discounting or risk taking in healthy adults. Experimental and Clinical Psychopharmacology, 14, 190-198. doi: 10.1037/1064-1297.14.2.190.

Adan, A. (2012). Impulsividad funcional y disfuncional en jóvenes con consumo intensivo de alcohol (binge drinking). Adicciones, 24, 17-22.

Albein-Urios, N., Martínez-González, J. M., Lozano, O., Clark, L., y Verdejo-García, A. (2012). Comparison of impulsivity and working memory in cocaine addiction and pathological gambling: Implications for cocaine-induced neurotoxicity. Drug and Alcohol Dependence, 126, 1-6. doi: 10.1016/j.drugalcdep.2012.03.008.

American Psychiatric Association, APA (2002). DSM-IV-TR. Manual diagnóstico y estadístico de los trastornos mentales. Texto revisado. Barcelona: Masson.

Arce, E., y Santisteban, C. (2006). Impulsivity: a review. Psicothema, 18, 213-220.

Balconi, M., Finocchiaro, R., y Campanella, S. (2014). Reward-sensitivity, decisional bias and metacognitive deficits in cocaine drug addiction. En 30th International Congress of Clinical Neurophysiology (pp. 1022-1022). International Federation of Clinical Neurophysiology. 
Barker, M. J., Greenwood, K. M., Jackson, M., y Crowe, S. F. (2004). Cognitive effects of long-term benzodiazepine use. CNS Drugs, 18, 37-48. doi:10.2165/00023210200418010-00004.

Bijttebier, P., Beck, I., Claes, L., y Vandereycken, W. (2009). Gray's Reinforcement Sensitivity Theory as a framework for research on personality-psychopathology associations. Clinical Psychology Review, 29, 421-430. doi:10.1016/j.cpr.2009.04.002.

Billieux, J., Rochat, L., Ceschi, G., Carré, A., Offerlin-Meyer, I., Defeldre, A. C., ... Van der Linden, M. (2012). Validation of a short French version of the UPPS-P impulsive behavior scale. Comprehensive Psychiatry, 53, 609-615. doi:10.1016/j.comppsych.2011.09.001.

Bravo de Medina, R., Echeburúa, E., y Aizpiri, J. (2007). Psychopathological symptoms and personality traits in alcohol-dependent patients: a comparative study. Adicciones, 19, 373-381.

Bravo de Medina, R., Echeburúa, E., y Azpiri, J. (2010). Psychopathological symptoms and personality traits in young adult cannabis-dependent treatment seekers: A comparative study. Adicciones, 22, 245-251.

Cano-Cervantes, G. J., Araque-Serrano, F., y Cándido-Ortiz, A., (2011). Addiction, Impulsivity And Temporal Curves Of Desire. Adicciones, 23, 141-148.

Carlson, S. R. y Pritchard, A. A. (2013). Externalizing behavior, the UPPS-P Impulsive Behavior scale and Reward and Punishment Sensitivity. Personality and Individual Differences, 54, 202-207. doi:10.1016/j.paid.2012.08.039.

Carroll, A., Hemingway, F., Bower, J., Ashman, A., Houghton, S., y Durkin, K. (2006). Impulsivity in juvenile delinquency: Differences among early-onset, late-onset, and non-offenders. Journal of Youth and Adolescence, 35, 517527. doi:10.1007/s10964-006-9053-6.

Casares-López, M. J., González-Menéndez, A., Bobes-Bascarán, M. T., Secades, R., Martínez-Cordero, A., y Bobes, J. (2011). Necesidad de evaluación de la patología dual en contexto penitenciario. Adicciones, 23, 37-44.

Caseras, X., Avila, C., y Torrubia, R. (2003). The measurement of individual differences in behavioural inhibition and behavioral activation Systems: a comparison of personality scales. Personality and Individual Differences, 34, 999-1013. doi:10.1016/S0191-8869(02)00084-3.

Cortés Tomás, M. T., Giménez Costa, J. A., Motos Sellés, P. y Cadaveira Mahía, F. (2014). The importance of expectations in the relationship between impulsivity and binge drinking among university students. Adicciones, 26, 134-145.

Cyders, M. A., y Smith, G. T. (2007). Mood-based rash action and its components: Positive and negative urgency. Personality and Individual Differences, 43, 839-850. doi:10.1016/j.paid.2007.02.008.

Cyders, M. A., Smith, G. T., Spillane, N. S., Fischer, S., Annus, A. M., y Peterson, C. (2007). Integration of impul- sivity and positive mood to predict risky behavior: Development and validation of a measure of positive urgency. Psychological Assessment, 19, 107-118. doi:10.1037/10403590.19.1.107.

Cyders, M. A., y Coskunpinar, A. (2011). Measurement of constructs using self-report and behavioral lab tasks: Is there overlap in nomothetic span and construct representation for impulsivity? Clinical Psychology Review, 31, 965-982. doi:10.1016/j.cpr.2011.06.001.

Dassanayake, T. L., Michie, P. T., Jones, A., Carter, G., Mallard, T., y Whyte, I. (2012). Cognitive impairment in patients clinically recovered from central nervous system depressant drug overdose. Journal of Clinical Psychopharmacology, 32, 503-510. doi:10.1097/ JCP.0b013e31825d6ddb.

Dawe, S., y Loxton, N. J. (2004). The role of impulsivity in the development of substance use and eating disorders. Neuroscience and Biobehavioral Reviews, 28, 343-351. doi:10.1016/j.neubiorev.2004.03.007.

Dawe, S., Gullo, M. J., y Loxton, N. J. (2004). Reward drive and rash impulsiveness as dimensions of impulsivity: implications for substance misuse. Addictive Behaviors, 29, 1389-1409. doi:10.1016/j.addbeh.2004.06.004.

Derefinko, K., DeWall, C. N., Metze, A. V., Walsh, E. C., y Lynam, D. R. (2011). Do different facets of impulsivity predict different types of aggression?. Aggressive Behav ior, 37, 223-233. doi:10.1002/ab.20387.

Dissabandara, L O., Loxton, N. J., Dias, S. R., Daglish, M., y Stadlin, A. (2012). Testing the fear and anxiety distinction in the BIS/BAS scales in community and heroin-dependents samples. Personality and Individual Differences, 52, 888-892. doi:10.1016/j.paid.2012.01.023.

Dissabandara, L. O., Loxton, N. J., Dias, S. R., Dodd, P. R., Daglish, M., y Stadlin, A. (2014). Dependent heroin use and associated risky behaviour: The role of rash impulsiveness and reward sensitivity. Addictive Behaviors, 39, $71-$ 76. doi:10.1016/j.addbeh.2013.06.009.

Dougherty, D. M., Mathias, C .W., Marsh-Richard, D. M., Nouvion, S. O., y Dawes, M. A. (2008). Distinctions in Behavioural Impulsivity: Implications for Substance Abuse Research. Addictive Disorders and Their Treatment, 8, 61-73. doi: 10.1097/ADT.0b013e318172e488.

Ersche, K. D., Roiser, J. P., Clark, L., London, M., Robbins, T. W., y Sahakian, B. J. (2005). Punishment induces risky decision-making in methadone-maintained opiate users but not in heroin users or healthy volunteers. Neuropsychopharmacology, 30, 2115-2124. doi:10.1038/ sj.npp.1300812.

Evenden, J. L. (1999). Varieties of impulsivity. Psychopharmacology, 146, 348-361. doi:10.1007/PL00005481.

Fernández-Serrano, M. J., Moreno-López, L., Pérez-García, M., Viedma-del Jesús, M. I., Sánchez-Barrera, M. B., y Verdejo-García, A. (2011). Negative mood induction normalizes decision making in male cocaine depen- 
dent individuals. Psychopharmacology, 217, 331-339. doi: 10.1007/s00213-011-2288-2.

Fernández-Sobrino, A. M., Fernandez-Rodriguez, V., y López-Castro, J. (2009). Consumo de benzodiacepinas en una muestra de pacientes en Programa de Tratamiento con Derivados Opiáceos (PTDO). Adicciones, 21, 143-146.

Franken, I. H. A., y Muris, P. (2006). Gray's impulsivity dimension: A distinction between reward sensitivity versus rash impulsiveness. Personality and Individual Differences, 40, 1337-1347. doi:10.1016/j.paid.2005.11.016.

Franken, I. H. A., Muris, P., y Georgieva, I. (2006). Gray's model of personality and addiction. Addictive Behaviors, 31, 399-403. doi:10.1016/j.addbeh.2005.05.022.

Gordon, V., y Egan, V. (2011). What self-report impulsivity measure best postdicts criminal convictions and prison breaches of discipline? Psychology, Crime and Law, 17, 305-318. doi:10.1080/10683160903203946.

Gray, J. A., y McNaughton, N. (2000). The neuropsychology of anxiety: An enquiry into the functions of the septo-hippocampal system (2nd ed.). Oxford, UK: Oxford University Press.

Gullo, M.J., Loxton, N. J., y Dawe, S. (2014). Impulsivity: Four ways five factors are not basic to addiction. Addictive Behaviors, 39, 1547-1556 doi:10.1016/j.addbeh.2014.01.002.

Jones, J. D., Mogali, S., y Comer, S. D. (2012). Polydrug abuse: a review of opioid and benzodiazepine combination use. Drug and Alcohol Dependence, 125, 8-18. doi:10.1016/j.drugalcdep.2012.07.004.

Lane, S. D., Tcheremissine, O. V., Lieving, L. M., Nouvion, S., y Cherek, D. R. (2005). Acute effects of alprazolam on risky decision making in humans. Psychopharmacology, 181, 364-373. doi:10.1007/s00213-005-2265-8.

Loxton, N. K., Wan, V. L. N., Hob, A. M. C., Cheungd, B. K. L., Tamb, N., Leung, F. Y. K., y Stadlin, A. S. (2008a). Impulsivity in Hong Kong-Chinese club-drug users. Drug and Alcohol Dependence, 95, 81-89. doi:10.1016/j.drugalcdep.2007.12.009.

Loxton, N. J., Nguyen, D., Casey, L. y Dawe, S. (2008b). Reward drive, rash impulsivity and punishment sensitivity in problem gamblers. Personality and Individual Differences, 45, 167-173. doi:10.1016/j.paid.2008.03.017.

Lykken, D. T. (1995). The antisocial personalities. Hillsdale, NJ: Erlbaum

Lyvers, M., Duff, H., Basch, V., y Edwards, M. S. (2012). Rash impulsiveness and reward sensitivity in relation to risky drinking by university students: Potential roles of frontal systems. Addictive Behaviors, 37, 940-946. doi:10.1016/j. addbeh.2012.03.028.

Mathias, C. W., Marsh-Richard, D. M., y Dougherty, D. M. (2008). Behavioral measures of impulsivity and the law. Behavioral Sciences and the Law, 26, 691-707. doi:10.1002/ bsl.841.

Michel, L., y Lang, J. P. (2003). Benzodiazepines and forensic aspects. L'Encephale, 29, 479-485.
Miller, J. D., Zeichner, A., y Wilson, L. F. (2012). Personality correlates of aggression: evidence from measures of the five-factor model, UPPS model of impulsivity, and BIS/BAS. Journal of Interpersonal Violence, 27, 2903-2919. doi:10.1177/0886260512438279 .

Mintzer, M. Z., y Stitzer, M. L. (2002). Cognitive impairment in methadone maintenance patients. Drug and Alcohol Dependence, 67, 41-51. doi:10.1016/S03768716(02)00013-3.

Mitchell, J. T., Kimbrel, N. A., Hundt, N. E., Cobb, A. R. Nelson-Gray, R. O. y Lootens, C. M. (2007). An Analysis of Reinforcement Sensitivity Theory and the Five-Factor Model. European Journal of Personality, 21, 869-887. doi:10.1002/per.644.

Moshier, S. J., Ewen, M., y Otto, M. W. (2013). Impulsivity as a moderator of the intention-behavior relationship for illicit drug use in patients undergoing treatment. Addictive Behaviors, 38, 1651-1655. doi: 10.1016/j.addbeh.2012.09.008.

Motos, P., Cortés Tomás, M. T., Giménez Costa, J. A., y Cadaveira Mahía, F. (2015). Predictors of weekly alcohol drinking and alcohol-related problems in binge-drinking undergraduates. Adicciones, 27, 119-131.

Navas, J. F., Torres, A., Cándido, A., y Perales, J. C., (2014). ¿'Nada'? o 'un poco'? ¿'Mucho' o 'demasiado'? La impulsividad como marcador de gravedad en niveles problemático y no problemático de uso de alcohol e Internet. Adicciones, 26, 159-167.

Nielsen, D. A. Ho, A., Bahl, A., Varma, P., Kellogg, S., Borg, L., y Kreek, M. J. (2012). Former heroin addicts with or without a history of cocaíne dependence are more impulsive than controls. Drug and Alcohol Dependence, 124, 113-120. doi: 10.1016/j.drugalcdep.

Observatorio Europeo de las Drogas y las Toxicomanías (EMCDDA) (2013). Informe Europeo sobre Drogas

Pattij, T., y De Vries, T. J. (2013). The role of impulsivity in relapse vulnerability. Current Opinion in Neurobiology, 23, 700-705. doi.org/10.1016/j.conb.2013.01.023.

Perales, J. C., Verdejo-García, A., Moya, M. I., Lozano, O., y Pérez-García, M. (2009). Bright and dark sides of impulsivity: Performance of individuals with high and low trait impulsivity on neuropsychological tasks. Journal of Clinical and Experimental Neuropsychology, 31, 927-944. doi: 10.1080/13803390902758793.

Perry, J. L., y Carroll, M. E. (2008). The role of impulsive behaviour in drug abuse. Psychopharmacology ,200, 1-26. doi: 10.1007/s00213-008-1173-0.

Prosser, J., Cohen, L. J., Steinfeld, M., Eisenberg, D., London, E. D., y Galynker, I. I. (2006). Neuropsychological functioning in opiate-dependent subjects receiving and following methadone maintenance treatment. Drug and Alcohol Dependence, 84, 240-247. doi:10.1016/j.drugalcdep.2006.02.006.

Ratchford, M., y Beaver, K. M. (2008). Neuropsychological deficits, low self-control, and delinquent in- 
volvement toward a biosocial explanation of delinquency. Criminal Justice and Behavior, 36, 147-162. doi:10.1177/0093854808325967.

Robinson, T. E., y Berridge, K. C. (1993). The neural basis of drug craving: an incentive-sensitization theory of addiction. Brain Research Reviews, 18, 247-291. doi:10.1016/0165-0173(93)90013-P.

Secretaría General de Instituciones Penitenciarias. Ministerio del Interior (2013). Informe General 2012

Smith, G. T., Fisher, S., Cyders, M. A., Annus, A. M., Spillane, N. S., y McCarthy, D. M. (2007). On the Validity and Utility of Discriminating Among Impulsivity-Like Traits. Assesment, 14, 155-170. doi:10.1177/1073191106295527.

Staiger, P. K., Kambouropoulos, N., y Dawe, S. (2007). Should personality traits be considered when refining substance misuse treatment programs?. Drug and Alcohol Review, 26, 17-23. doi:10.1080/09595230601036952.

Stautz, K., y Cooper, A. (2013). Impulsivity-related personality traits and adolescent alcohol use: a meta-analytic review. Clinical Psychology Review, 33, 574-592. doi:10.1016/j.cpr.2013.03.003.

Stewart, S. A. (2005). The effects of benzodiazepines on cognition. Journal of Clinical Psychiatry, 66, S9-S13.

Subdirección General de Sanidad Penitenciaria (2007). Estudio sobre salud mental en el medio penitenciario.

Torrubia, R., Avila, C., Moltó, J., y Caseras, X. (2001). The Sensitivity to Punishment and Sensitivity to Reward Questionnaire (SPSRQ) as a mesure of Gray's anxiety and impulsivity dimensions. Personality and Individual $\mathrm{Di}$ fferences, 31, 837-862.

Verdejo-García, A., Sánchez-Fernández, M. M., Alonso-Maroto, L. M., Fernández-Calderón, F., Perales, J. C., Lozano, O., y Pérez-García, M. (2010a). Impulsivity and executive functions in polysubstance-using rave attenders. Psychopharmacology, 210, 377-392. doi: 10.1007/S00213010-1833-8.

Verdejo-García, A., Lozano, O., Moya, M. I., Alcázar, M. A., y Pérez-García, M. (2010b). Psychometric Properties of a Spanish Version of the UPPS-P Impulsive Behavior Scale: Reliability, Validity and Association with Trait and Cognitive Impulsivity. Journal of Personality Assessment, 92, 70-77. doi:10.1080/00223890903382369.

Whiteside, S. P., y Lynam, D. R. (2001). The Five Factor Model and impulsivity: using a structural model of personality to understand impulsivity. Personality and Individual Differences, 30, 669-689. doi:10.1016/S01918869(00)00064-7.

Zermatten, A., Van der Linden, M., d'Acremont, M., Jermann, F., y Bechara, A. (2005). Impulsivity and decision making. The Journal of Nervous and Mental Disease, 193, 647-650. doi:10.1097/01.nmd.0000180777.41295.65. 